\title{
A universal description of temporal and lateral distributions of ground particles in extensive air showers
}

\author{
Maximo Ave, Markus Roth* and Alexander Schulz \\ Institut für Kernphysik, Karlsruhe Institute of Technology (KIT), Germany \\ E-mail: maximo.ave.personal@gmail.com, markus.roth@kit.edu, \\ alexander.schulz@kit.edu
}

Extensive air showers are traditionally described with phenomenological models - often called Lateral Distribution Functions (LDFs) - of the density of particles at the ground and derived quantities. The concept of air shower universality aims at a deeper understanding of the shower development in the atmosphere by taking into account physical properties of different types of secondary particles. Our extended model is based on the well known universal behaviour of the electromagnetic as well as of the muonic component and of its accompanying electromagnetic halo. A fourth component of electromagnetic particles from pion decays close to the observation level is considered in addition. Eventually the model allows for a description of particle distributions at observation level as a function of a few macroscopic quantities: the total energy $E$, the depth of the shower maximum $X_{\max }$, the muon content $N_{\mu}$ and the geometry of the shower. The pure electromagnetic component is determined by $E$ and $X_{\max }$ while differences between hadronic interaction models and primary particles are absorbed in the muon scale, affecting the three remaining components. We will detail the basic concepts of the extended universal description of air showers and describe the application using the detector response of the water-Cherenkov detector array of the Pierre Auger Observatory as an example. Both, the signal response of particles and their time of arrival in the detectors are accounted for in the reconstruction. The universal parametrizations of the components allow us to estimate $X_{\max }$ and $N_{\mu}$ event-by-event solely based on the measured footprint of the air shower at observation level. The shower maximum is reconstructed with a resolution of $30-50 \mathrm{~g} / \mathrm{cm}^{2}$ depending on energy and zenith angle of the shower. The applicability of the method, limitations and model dependence is discussed.

The 34th International Cosmic Ray Conference,

30 July- 6 August, 2015

The Hague, The Netherlands

\footnotetext{
*Speaker.
} 


\section{Introduction}

The fact that the average properties of an extensive air shower can, to a large extent, be described in terms of energy and shower age only is called shower universality, see [1] and Refs. within. To a first approximation, there is no direct dependence on the primary mass nor zenith angle, which is a very remarkable result. The vast number of interactions in an air shower, its overall shape, as well as the time profiles of particles reaching the ground, can be described very well with very few measurable quantities. In literature, universality has been described for the electromagnetic component of showers $[1,2,3,4,5]$. The concept can be extended to hadronic showers by introducing one additional parameter, the muon scale $N_{\mu}[6,7,8]$. The result is a model that describes showers initiated by protons, nuclei up to iron as well as pho-

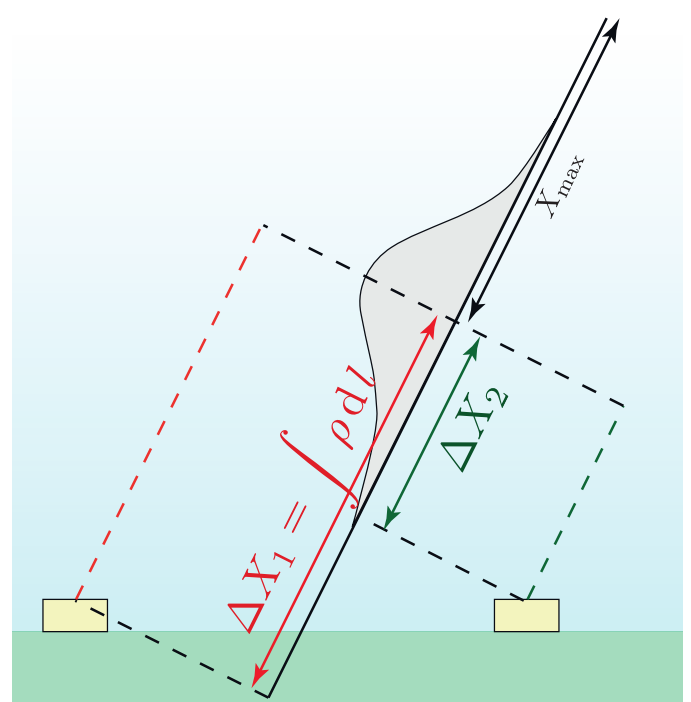

Figure 1: The distance from ground to the shower maximum $(\Delta X)$. The density of the atmosphere is integrated from $X_{\max }$ to the projected position of the detector on the shower axis. ton showers using only a few parameters: $E$, the shower geometry, the distance to $X_{\max }, \Delta X$ (for definition see fig. 1), and $N_{\mu}$. The main advantage of this method is that it exploits all experimentally collected information of an air shower for deriving the physics observables. In this sense a universality-based reconstruction can be considered as a very advanced multivariate analysis of the shower data that employs parameterized physics relations to combine the different measured quantities. A weakness of the universality approach is that is has systematic uncertainties that are difficult to control. The parameterization of the universality relations between the energy, shower age (i.e. $\mathrm{X}_{\max }$ ), and muon number, and the different signal components at ground (for different lateral distances), can only be derived from libraries of simulated showers and depends to some degree on the hadronic interaction models used for the simulations. the high energy interaction model quantities.

\section{Signal model}

To fully exploit the universality features of air showers, four shower components have to be introduced: (a) the muonic component $(\mu)$, (b) the electromagnetic component stemming from muon interactions and muon decay $(e \gamma(\mu))$, (c) the purely electromagnetic component $(e \gamma)$, and (d) the electromagnetic component from low-energy hadrons ( $e \gamma($ had $)$, the collimated beam component). Splitting the electromagnetic component into component (c), originating from the decay products of high energy $\pi^{0}$ that have been produced in the first generations of hadronic interactions in a shower, and component (d) which is stemming from hadronic interactions at low energy taking place close to the individual detectors, allows us to include the correlation between muons and electromagnetic particles arising from such low energy interactions. Within this framework, 

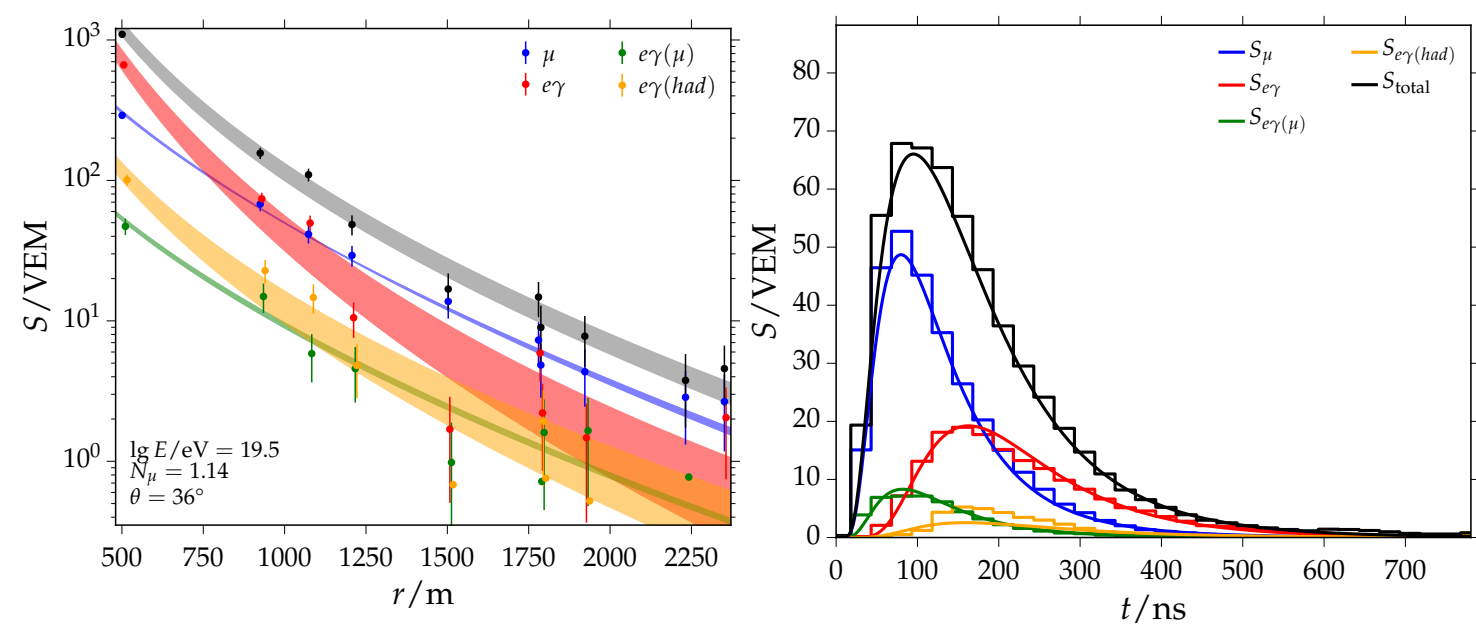

Figure 2: (Left) The lateral shape of the predicted signal for a proton shower at $10^{19.5} \mathrm{eV}$. The upper band indicates the model prediction based on the fitted parameters. Round markers refer to the total simulated signal. The lower bands show the prediction for the four signal components compared to the simulated values. The size of the signal asymmetry is indicated by the width of the bands: The upper and lower boundaries show the extreme case of $\Psi=0^{\circ}$ and $180^{\circ}$. The large asymmetries of the electromagnetic compared to the muonic components becomes apparent. (Right) The PMT response of the four signal components after digitization in the FADC and VEM calibration for station closest to the shower core of the same event. The delay of the electromagnetic part of the shower w.r.t. the muonic component is apparent. Model predictions for the different particle types are plotted in comparison.

the systematic errors in the prediction of the signal response of a water-Cherenkov detector of the Pierre Auger type $[9,10]$ are well below $5 \%$ over the whole ranges of energy and zenith angle considered for this study. The model includes all asymmetries in the ground signal. Effects independent of the detector are the attenuation of the electromagnetic shower in the atmosphere and the screening of a part of the shower by the ground. Furthermore, the detector geometry introduces asymmetries in the angular distribution of incident secondary particles and in the average track length of particles that pass the detector volume.

\section{Time model}

The concept of shower universality is extended to the time distribution of particles on ground. As the secondary particles pass the detector volume they create Cherenkov radiation that is collected by photomultipliers and digitized by a flash ADC at $40 \mathrm{MHz}$. All relevant physical and electronic processes are simulated with the Offline framework [11]. The simulation is based on a library of more than 8000 CORSIKA showers [12] with different energies $\left(10^{18} \mathrm{eV}, 10^{18.5} \mathrm{eV}\right.$, $10^{19} \mathrm{eV}, 10^{19.5} \mathrm{eV}$ and $\left.10^{20} \mathrm{eV}\right)$ and various zenith angles $\left(0^{\circ}, 37^{\circ}, 48^{\circ}, 55^{\circ}\right.$ and $\left.60^{\circ}\right)$. The high energy hadronic interactions are simulated with the model QGSJet-II.03 [13]. An example of the simulated time response after digitization is shown in fig. 2 (right). The $25 \mathrm{~ns}$ FADC traces of all four signal components can be modeled precisely by a log-normal function with four parameters 

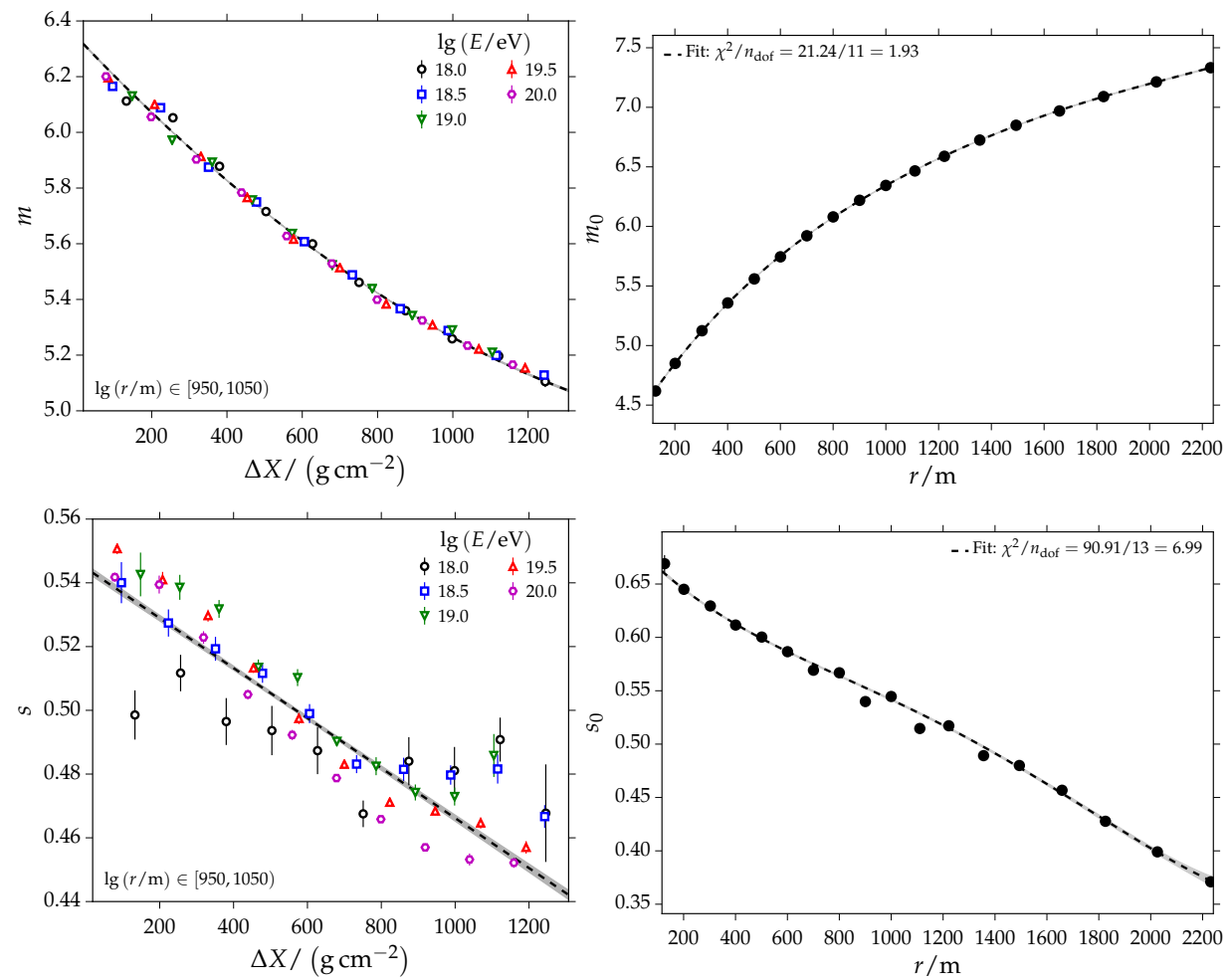

Figure 3: The parameters $m$ (upper panel) and $s$ (lower panel) of the log-normal for the purely muonic component. The left plots include DX dependencies for a certain radial slice and different primary energies. The main amplitude of the radial dependence is shown in the right plots.

$A, m, s, t_{0}$ :

$$
f\left(t, t_{0}, m, s\right)=\left\{\begin{array}{cc}
A \cdot \frac{1}{\left(t-t_{0}\right) \cdot s \cdot \sqrt{2 \pi}} e^{-\frac{\left(\ln \left(t-t_{0}\right)-m\right)^{2}}{2 s^{2}}} & \text { for } t>t_{0} \\
0 & \text { for } t \leq t_{0}
\end{array}\right.
$$

The time offset $t_{0}$ is given by the physically earliest possible time for a particle of a certain type to arrive at a detector at the position $\vec{P}_{\text {detector }}$. It is given by its distance to the point of first interaction $\vec{P}_{\text {first }}$ and the distance $r$ to the shower axis $t_{0}=\frac{r^{2}}{2 c}\left(\left|\vec{P}_{\text {detector }}-\vec{P}_{\text {first }}\right|\right)^{-1}$, where $t_{0}$ is fixed for each individual time trace, while the parameters $m$ and $s$ are fitted. The normalization $A$ is not considered further since it is taken into account with the signal model. $m$ and $s$ are parametrized as a function of $\Delta X, r, \psi, \theta$ and $\lg E$ with $\psi$ being the azimuth angle in shower plane coordinates. The general model for the description of the parameters $m$ and $s$ reads:

$$
\begin{aligned}
f_{\mathrm{m}, \mathrm{s}}(\Delta X, \psi, \theta, E) & =f_{\Delta X}(\Delta X)+f_{\mathrm{geo}}(\theta, \psi, \Delta X)+f_{\lg E}(\lg E, \Delta X) \quad \text { with } \\
f_{\Delta X}(\Delta X) & =a_{\Delta X}+\Delta X_{\mathrm{ref}}\left(b_{\Delta X}+c_{\Delta X} \Delta X_{\mathrm{ref}}\right) \\
f_{\mathrm{geo}}(\theta, \psi, \Delta X) & =\sin \theta\left(a_{\mathrm{geo}} \cos \psi+b_{\mathrm{geo}} \Delta X_{\mathrm{ref}}\right) \\
f_{E}(\lg E, \Delta X) & =\lg E_{\mathrm{ref}}\left(a_{\lg E}+b_{\lg E} \Delta X_{\mathrm{ref}}\right) \\
\Delta X_{\mathrm{ref}} & =\Delta X /\left(750 \mathrm{~g} \mathrm{~cm}^{-2}\right) \quad \text { and } \\
\lg E_{\mathrm{ref}} & =\lg (E / \mathrm{eV})-19 .
\end{aligned}
$$



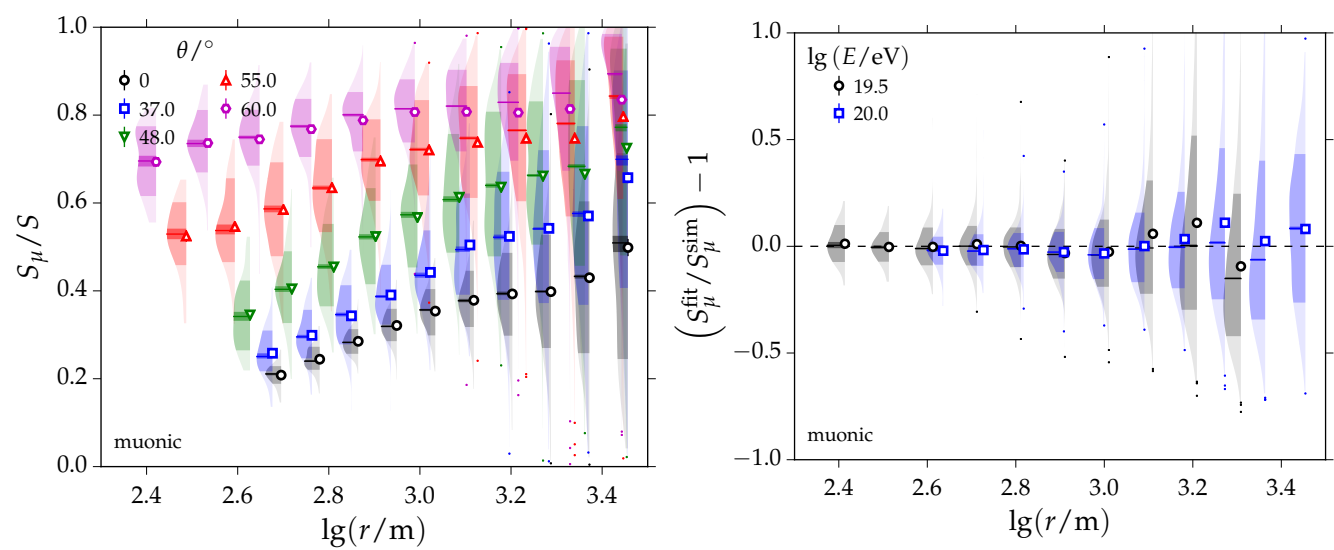

Figure 4: (Left) Ratios of the purely muonic signals with respect to the total signal as a function of the distance to the shower axis. Full distributions for different zenith angles are plotted. (Right) Relative ratio of fitted and simulated muonic signal as a function of distance to the shower core for two different energies.

The model is obtained separately for all four signal components. Depending on particle component and parameter some quantities in the above model are found to be zero. The fitting procedure is divided into two steps. Firstly, a modelling of all dependencies $f_{\mathrm{m}, \mathrm{s}}(\Delta X, \psi, \theta, E)$ except the radial one and, secondly, a fit of the remaining radial dependencies (not given here). Examples of fits for the purely muonic component are given in fig. 3. The shape parameters $(m, s)$ have a small dependence on the hadronic interaction model and primary particle type. For the derivation of the time model, the average of all available showers is taken. The systematic effects introduced in the reconstruction by ignoring those non-universal features have been studied and found to be small.

\section{Reconstruction algorithm}

The models described above give a complete description of the expected time-dependent signal in detectors at ground level. This is used to fit the shower geometry, $\mathrm{N}_{\mu}$ and $\mathrm{X}_{\max }$ on a single event basis using the time traces of a set of surface detectors. The reconstruction algorithm is implemented in the form of a log likelihood maximization:

$$
\begin{aligned}
\ln L_{\text {total }} & =f\left(\mathrm{~N}_{\mu}, E, X_{\max }, X_{0}, \vec{r}_{\mathrm{BC}}, t_{\text {core }}, \theta, \phi\right) \\
& =\sum_{\text {detectors k }} \ln L_{\text {shape }}^{k} \\
& +\sum_{\text {detectors i }} \ln L_{\text {signal }}^{i}+\ln L_{\text {start }}^{i}
\end{aligned}
$$

The core is specified in barycentric coordinates $\vec{r}_{\mathrm{BC}}$ (the barycenter is the signal-weighted average of all detector positions) and the time $t_{\text {core }}$ when the shower axis intersects with the ground surface. The shower axis is given in spherical coordinates $\theta, \phi$. If the time trace of a detector has at least 5 bins above 0.2 VEM, it is included in the bin-by-bin likelihood (shape fit). All remaining detectors contribute with their total signal and start time. In a water-Cherenkov detector, the muonic and electromagnetic contributions to the signal cannot be disentangled completely. Furthermore, the signal components have a non-trivial radial dependence as depicted in fig. 4 (left). 

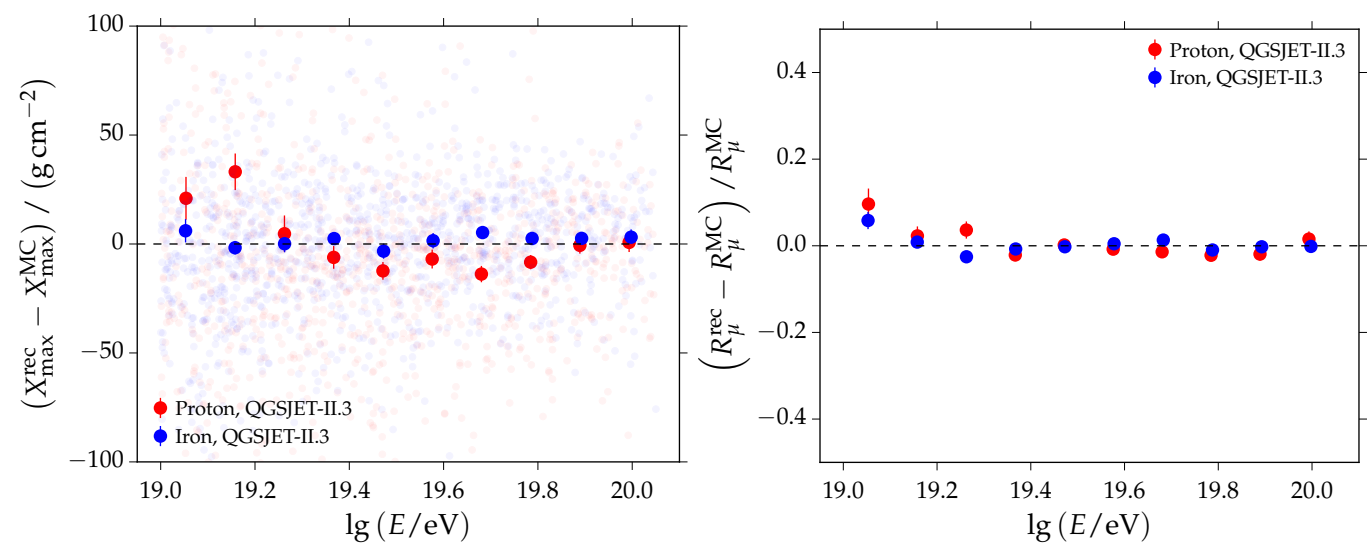

Figure 5: Average bias of the reconstructed $\mathrm{X}_{\max }$ (left panel) and $\mathrm{R}_{\mu}$ (right panel) for proton and iron showers.

This leads to a correlation between energy and $\mathrm{N}_{\mu}$. For both, unbiased estimates can be obtained on a single event basis at the expense of a comparatively large spread. Therefore, the energy is fixed. In simulated events, the true energy is used. For real events, the energy would be obtained from a calibration curve based on events where both fluorescence and surface detector data are available. The total expected signal of a component $c$ in a detector at $R_{\mathrm{SP}}, \psi_{\mathrm{SP}}$ in shower plane coordinates is $S_{c}=f\left(\mathrm{~N}_{\mu}, E, \Delta X, \theta, R_{\mathrm{SP}}, \psi_{\mathrm{SP}}\right)$. The total expected signal reads as $S_{\text {tot }}=\sum_{c} S_{c}$. The expected normalized signal at the time $t_{\mathrm{PF}}$ referred to the arrival time of the plane front of a component $c$ is given by $s_{c}=f\left(\Delta X, \Delta X_{0}, \theta, R_{\mathrm{SP}}, \psi_{\mathrm{SP}}, t_{\mathrm{PF}}\right)$. The total expected signal at the time $t_{\mathrm{PF}}$ is a weighted sum of the component signals and time shapes: $S_{\mathrm{tot}}\left(t_{\mathrm{PF}}\right)=\sum_{c} S_{c} \cdot s_{c}\left(t_{\mathrm{PF}}\right)$ Using the standard normal distribution $f_{\mathrm{G}}(x, \mu, \sigma)=\frac{1}{\sqrt{2 \pi} \sigma} \exp \left(-(x-\mu)^{2} / 2 \sigma^{2}\right)$ the bin-by-bin time contribution of a detector $k$ with the measured signal $S_{k}^{m}$ in the bin $m$ to the likelihood reads as $\ln L_{\text {shape }}^{k}=\sum_{\text {bins } m} \ln f_{G}\left[S_{k}^{m}, S_{\text {tot }}^{m}\left(t_{\mathrm{PF}}\right), \sigma\left(S_{\text {tot }}^{m}\right)\right]$. This is essentially a chi-square contribution of the traces to the time model summed over all selected detectors. The contribution of the total integrated signal is determined by $\ln L_{\text {signal }}^{i}=\ln f_{G}\left[S^{m}, S_{\text {tot }}, \sigma\left(S_{\text {tot }}\right)\right]$. Again, this corresponds to a chi-square contribution written in the form of a log-likelihood. The start time of the signal determines the arrival direction of the shower as well as the curvature of the shower front. The p.d.f. $f_{1}(t)$ of the start time is obtained from the time model $f(t)$ and the number of particles $n$ in the detector using an extreme value transformation: $f_{1}\left(t_{\text {start }}\right)=n\left(1-\int_{0}^{t_{\text {start }}} f\left(t^{\prime}\right) d t^{\prime}\right)^{n-1} f\left(t_{\text {start }}\right)$. It is assumed that the early part of the shower front consists mainly of muons and thus the time model for the pure muonic component may be used. $n$ is obtained from the expected muonic signal and the zenith-dependent track length in the detector. The likelihood contribution of a detector $i$ reads as $\ln L_{\text {start }}^{i}=\ln f_{1}\left(t_{\text {start }}^{i}\right)$.

\section{Reconstruction results}

As an example, a simulated proton shower with $E=10^{19.5} \mathrm{eV}$ and $\theta=36^{\circ}$ was chosen to visualize the reconstruction. Since the time structure and the signals of stations at different $R_{\mathrm{SP}}, \psi_{\mathrm{SP}}$ in the shower plane contribute to the result, some projection has to be chosen. In fig. 2 (left), the lateral shape of the fitted model is shown. The prediction of the component signal is in good 

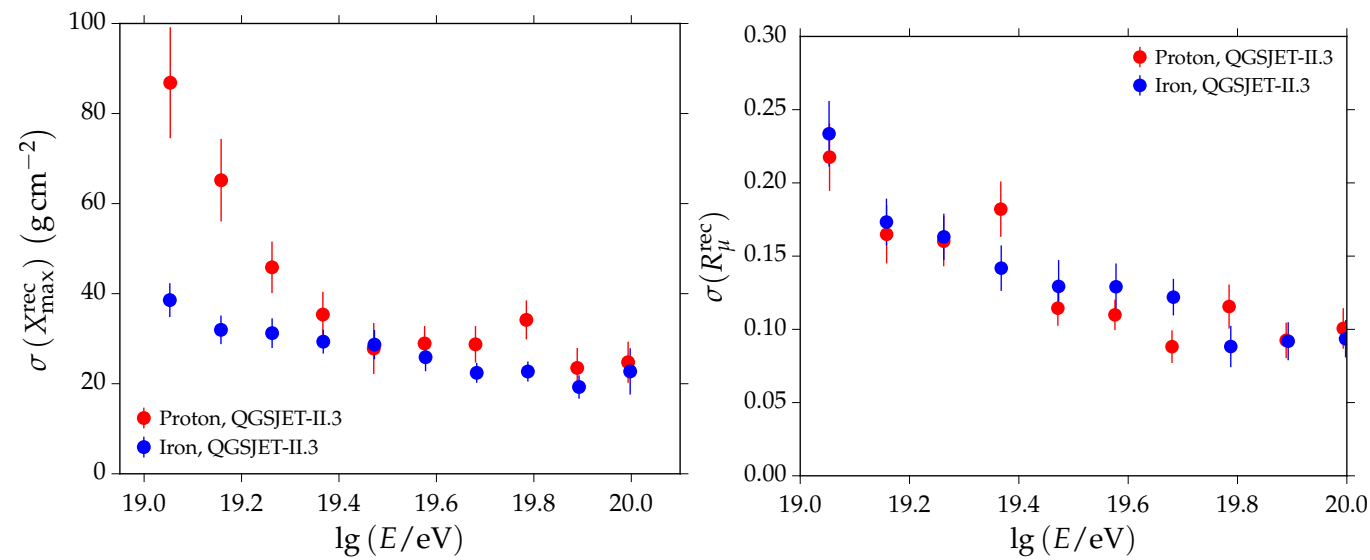

Figure 6: Spread of the reconstructed $\mathrm{X}_{\max }$ (left panel) and $\mathrm{R}_{\mu}$ (right panel) for proton and iron showers.

agreement with the simulated values. The time traces of the station with highest signal is depicted in fig. 2 (right). Overall the simulation of recorded component traces (histograms) are in good agreement with the fitted component p.d.f.s (lines). The relative difference for the muonic component averaged over all events at two different energies is shown in fig. 4 (right), where an unbiased estimate is observed up to very large distances. An unbiased $\mathrm{X}_{\max }$ (see fig. 5 (left)) is reconstructed with this method with an energy-dependent resolution of $50 \mathrm{~g} \mathrm{~cm}^{-2}$ to $30 \mathrm{~g} \mathrm{~cm}^{-2}$. The spread in the reconstructed $X_{\max }$ is smaller for iron than for proton showers (see left panel of fig. 6). Apart from the energy, the resolution depends mainly on zenith angle. The energy dependence is obtained by marginalizing the two-dimensional distribution in $E$ and $\theta$. This may be done if the $\theta$-distribution in the shower library reflects the distribution in real data $(N(\theta) \propto \sin \theta \cos \theta)$. The mean expected $\mathrm{N}_{\mu}$ is given in relative units $\mathrm{R}_{\mu}$ w.r.t. proton showers simulated with QGSJet-II.03 and therefore $R_{\mu}^{\mathrm{p}}=1.0$. For iron, the expected value is $R_{\mu}^{\mathrm{Fe}}=1.3$. The resolution of $\mathrm{R}_{\mu}$ is shown in the right panel of fig. 6. It corresponds to $30 \%$ to $50 \%$ of the separation between proton and iron.

\section{Summary}

It is possible to describe the signal and time distribution of secondary particles on ground based on shower universality. The model presented in this note predicts the expected signal in a water-Cherenkov detector of Pierre Auger type. Based only on ground particle information, it is possible to reconstruct $X_{\max }$ and $\mathrm{N}_{\mu}$ and an unbiased core position. In a fluorescence measurement, the energy carried by muons and neutrinos is invisible. The calorimetric energy underestimates the true energy. If an estimate of the true energy is obtained by externals means, it is possible to determine the amount of the missing energy [14] based on shower universality on a single event basis. Nevertheless, these results are based on Monte Carlo parametrizations which eventuate in large systematic uncertainties and call for a significant step forward in a direct measurement of individual components of air-shower events. An independent determination of the number of muons is required for the full potential of these universality methods to be exploited. Such a measurement will also give a handle on better understanding of the systematic uncertainties. 
Acknowledgement It is our pleasure to thank our colleagues from the Pierre Auger Collaboration for many fruitful discussions. This work is supported in part by the Helmholtz Alliance for Astroparticle Physics HAP, which is funded by the Initiative and Networking Fund of the Helmholtz Association.

\section{References}

[1] P. Lipari, The Concepts of 'Age' and 'Universality' in Cosmic Ray Showers, Phys. Rev. D79 (2008) 063001, [arXiv:0809.0190].

[2] A. M. Hillas, Angular and energy distributions of charged particles in electron photon cascades in air, J. Phys. G8 (1982) 1461-1473.

[3] M. Giller, A. Kacperczyk, J. Malinowski, W. Tkaczyk, and G. Wieczorek, Similarity of extensive air showers with respect to the shower age, J. Phys. G31 (2005) 947-958.

[4] F. Nerling, J. Blümer, R. Engel, and M. Risse, Universality of electron distributions in high-energy air showers: Description of cherenkov light production, Astropart. Phys. 24 (2006) 421-437, [astro-ph/0506729].

[5] S. Lafebre, R. Engel, H. Falcke, J. Hörandel, T. Huege, J. Kuijpers, and R. Ulrich, Universality of electron-positron distributions in extensive air showers, Astropart. Phys. 31 (2009) 243-254, [arXiv:0902.0548].

[6] F. Schmidt, M. Ave, L. Cazon, and A. S. Chou, A Model-Independent Method of Determining Energy Scale and Muon Number in Cosmic Ray Surface Detectors, Astropart. Phys. 29 (2008) 355-365, [arXiv:0712.3750].

[7] M. Ave, R. Engel, J. Gonzalez, D. Heck, T. Pierog, and M. Roth, Extensive air shower universality of ground particle distributions, Proc. of 32nd Int. Cosmic Ray Conf., Beijing 2 (2011) 178-181.

[8] D. Maurel, M. Roth, and J. Gonzalez, Universality of the time structure of ground particle distributions and its application to the reconstruction of extensive air showers, in Proc. of 33rd Int. Cosmic Ray Conf., Rio de Janeiro, 2013.

[9] Pierre Auger Collaboration, A. Aab et al., The Pierre Auger Cosmic Ray Observatory, to appear in Nucl. Instrum. Meth. A (2015) [arXiv: 1502.0132].

[10] Pierre Auger Collaboration, I. Allekotte et al., The Surface Detector System of the Pierre Auger Observatory, Nucl. Instrum. Meth. A586 (2008) 409-420, [arXiv: 0712.2832].

[11] S. Argirò, S. Barroso, J. Gonzalez, L. Nellen, T. Paul, T. Porter, L. P. Jr., M. Roth, R. Ulrich, and D. Veberič, The offline software framework of the Pierre Auger Observatory, Nucl. Instrum. Meth. A580 (2007) 1485-1496.

[12] D. Heck, G. Schatz, T. Thouw, J. Knapp, and J. Capdevielle, CORSIKA: A Monte Carlo code to simulate extensive air showers, Forschungszentrum Karlsruhe Report FZKA (1998) 6019.

[13] S. Ostapchenko, Monte Carlo treatment of hadronic interactions in enhanced Pomeron scheme: I. QGSJET-II model, Phys. Rev. D83 (2011) 014018, [arXiv: 1010 . 1869].

[14] H. M. J. Barbosa, F. Catalani, J. A. Chinellato, and C. Dobrigkeit, Determination of the calorimetric energy in extensive air showers, Astropart. Phys. 22 (2004) 159-166, [astro-ph/ 0310234 ]. 